\title{
C6-substituted Analogs of 8-Azanebularine: Probes of an RNA-editing Enzyme Active Site
}

\author{
Olena Maydanovych and Peter A. Beal* \\ Department of Chemistry, University of Utah, Salt Lake City, Utah 84112 \\ beal@chem.utah.edu
}

Supporting Information 
General synthetic procedures. All reagents and solvents were purchased from Sigma/Aldrich or Fischer Scientific and were used without additional purification unless stated otherwise. Ethanol and acetonitrile were distilled from calcium hydride. Tetrahydrofuran was distilled from sodium metal and benzophenone. Reactions were performed under an atmosphere of dry nitrogen when anhydrous conditions were required. Solvents and liquid reagents were introduced by oven-dried micro syringes. Merck silica gel $60 \mathrm{~F}_{254}$ precoated plates were used for thin layer chromatography (TLC). Flash column chromatography was conducted using Mallinckrodt Baker silica gel 150 (60-200 mesh). Melting points were determined by open capillary method on MELTEMP II apparatus and are uncorrected. ${ }^{1} \mathrm{H} \mathrm{NMR},{ }^{13} \mathrm{C} \mathrm{NMR}$ and ${ }^{31} \mathrm{P}$ spectra were recorded at $300 \mathrm{MHz}, 75 \mathrm{MHz}$ and $121 \mathrm{MHz}$ respectively. The chemical shifts are reported as parts per million $(\delta)$ relative to the solvent peak. The chemical shifts for phosphorus NMR are reported in parts per million using $85 \%$ phosphoric acid as an external standard. The ${ }^{1} \mathrm{H}$ NMR data are reported as peak multiplicities: s for singlet; $\mathrm{d}$ for doublet; dd for doublet of doublets; $\mathrm{t}$ for triplet; brs for broad singlet; brm for broad multiplet; and m for multiplet. All HRFABMS analyses were performed on a Finnigan MAT 95 mass spectrometer. MALDI analysis was obtained from University of Utah Mass Spectrometry and Proteomics Core Facility on a Voyager-DE STR MALDI mass spectrometer.

$2^{\prime}, 3^{\prime}, 5^{\prime}$-Tri- $O$-acetyl-6-bromo-8-azanebularine (2) was prepared following a previously reported procedure. ${ }^{1}$

2',3',5'-Tri- $O$-acetyl-6-methyl-8-azanebularine (3). To a solution of 2',3',5'-tri- $O$ acetyl-6-bromo-8-azanebularine $(194.3 \mathrm{mg}, 0.42 \mathrm{mmol})$ and $\mathrm{Pd}\left(\mathrm{PPh}_{3}\right)_{4}(49.0 \mathrm{mg}, 0.042$ 
mmol) in NMP $(900 \mu \mathrm{L})$ under argon atmosphere was added $\mathrm{Sn}\left(\mathrm{CH}_{3}\right)_{4}(117.5 \mu \mathrm{L}, 0.85$ mmol). The reaction solution was stirred at $85{ }^{\circ} \mathrm{C}$ for $20 \mathrm{~h}$. The resulting mixture was diluted with EtOAc $(12 \mathrm{~mL})$, washed with water $(2 \times 5 \mathrm{~mL})$ and brine $(5 \mathrm{~mL})$. The organic portion was dried over $\mathrm{Na}_{2} \mathrm{SO}_{4}$, filtered, and concentrated under reduced pressure. Purification of crude product by flash column chromatography $\left(\mathrm{CH}_{2} \mathrm{Cl}_{2} / \mathrm{Hexanes}\right.$ 1:1 followed by $\mathrm{CH}_{2} \mathrm{Cl}_{2} / \mathrm{CH}_{3} \mathrm{OH}$ 99:1) gave a brown foam $(93.5 \mathrm{mg}$, 57\%). ${ }^{1} \mathrm{H}$ NMR $\left(\mathrm{CD}_{2} \mathrm{Cl}_{2}, 300 \mathrm{MHz}\right): \delta(\mathrm{ppm}) 9.03(\mathrm{~s}, 1 \mathrm{H}), 6.65(\mathrm{~d}, J=3.9 \mathrm{~Hz}, 1 \mathrm{H})$, 6.21-6.18 (m, 1H), 5.87-5.83 (m, 1H), 4.55-4.51 (m, 1H), 4.48-4.17 (m, 2H), 3.01(s, 3H), $2.14(\mathrm{~s}, 3 \mathrm{H}), 2.09(\mathrm{~s}, 3 \mathrm{H}), 2.02(\mathrm{~s}, 3 \mathrm{H}) .{ }^{13} \mathrm{C} \mathrm{NMR}\left(\mathrm{CD}_{2} \mathrm{Cl}_{2}, 75 \mathrm{MHz}\right): \delta(\mathrm{ppm})$ 170.9, 170.2, 170, 164.2, 156.9, 149.2, 136.5, 88.1, 81.3, 73.6, 71.4, 63.3, 21.0, 20.9, 20.8, 20.7. HRFABMS: calcd. for $\mathrm{C}_{16} \mathrm{H}_{20} \mathrm{~N}_{5} \mathrm{O}_{7}(\mathrm{M}+\mathrm{H})^{+}$394.1363, obsd. 394.1374.

2',3',5'-Tri- $\boldsymbol{O}$-acetyl-6-mercapto-8-azanebularine (4). To a solution of 2',3',5'-tri- $O$ acetyl-6-bromo-8-azanebularine $(150 \mathrm{mg}, 0.33 \mathrm{mmol})$ in freshly distilled ethanol $(4 \mathrm{~mL})$ was added thiourea $(500 \mathrm{mg}, 6.55 \mathrm{mmol})$ and reaction mixture was reacted at reflux for $17 \mathrm{~h}$. The resulting solution was concentrated in vacuo to dryness and crude product was purified by flash column chromatography $\left(\mathrm{CH}_{2} \mathrm{Cl}_{2} / \mathrm{CH}_{3} \mathrm{OH}\right.$ 99:1) to give a white foam (110 mg, 78\%). ${ }^{1} \mathrm{H}$ NMR $\left(\mathrm{CD}_{2} \mathrm{Cl}_{2}, 300 \mathrm{MHz}\right): \delta(\mathrm{ppm}) 8.69$ (s, $\left.1 \mathrm{H}\right), 7.68-7.65$ (brm, 1H), 6.33-6.29 (brm, 1H), 5.53-5.44 (m, 2H), 4.45-4.16 (m, 3H), $2.14(\mathrm{~s}, 3 \mathrm{H}), 2.12(\mathrm{~s}$, $3 \mathrm{H}), 2.1(\mathrm{~s}, 3 \mathrm{H}) .{ }^{13} \mathrm{C} \mathrm{NMR}\left(\mathrm{CD}_{2} \mathrm{Cl}_{2}, 75 \mathrm{MHz}\right): \delta(\mathrm{ppm}) 171.1,170.5,170.4,169.9$, 157.7, 156.5, 137.6, 83.9, 79.9, 79.7, 71.2, 63.9, 21.2, 20.9, 20.9. HRFABMS: calcd. for $\mathrm{C}_{15} \mathrm{H}_{18} \mathrm{~N}_{5} \mathrm{O}_{7} \mathrm{~S}(\mathrm{M}+\mathrm{H})^{+}$412.0928, obsd. 412.0936.

2',3',5'-Tri- $O$-acetyl-6-cyano-8-azanebularine (5). To a solution of 2',3',5'-tri- $O$-acetyl6-bromo-8-azanebularine $(350 \mathrm{mg}, 0.76 \mathrm{mmol})$ in freshly distilled acetonitrile $(8 \mathrm{~mL})$ 
were added tetraethylammoniumcyanide $(131 \mathrm{mg}, 0.84 \mathrm{mmol})$ followed by DABCO (94 $\mathrm{mg}, 0.84 \mathrm{mmol}$ ) at $0{ }^{\circ} \mathrm{C}$ and stirred at room temperature for $7 \mathrm{~h}$. The resulting mixture was concentrated in vacuo to dryness, re-dissolved in dichloromethane, washed with water $(2 \times 20 \mathrm{~mL})$, dried over $\mathrm{NaSO}_{4}$ and concentrated under reduced pressure. Obtained crude material was purified by flash column chromatography (Hexanes/Ethyl Ether 1:1) to produce a white foam $(188.7 \mathrm{mg}, 61 \%) .{ }^{1} \mathrm{H} \mathrm{NMR}\left(\mathrm{CD}_{2} \mathrm{Cl}_{2}, 300 \mathrm{MHz}\right): \delta(\mathrm{ppm}) 9.37$ $(\mathrm{s}, 1 \mathrm{H}), 6.74(\mathrm{~d}, J=3.9 \mathrm{~Hz}, 1 \mathrm{H}), 6.21-6.18(\mathrm{~m}, 1 \mathrm{H}), 5.84-5.80(\mathrm{~m}, 1 \mathrm{H}), 4.57(\mathrm{dd}, J=8.5$, $4.9 \mathrm{~Hz}, 1 \mathrm{H}), 4.47-4.19(\mathrm{~m}, 2 \mathrm{H}), 2.15(\mathrm{~s}, 3 \mathrm{H}), 2.09(\mathrm{~s}, 3 \mathrm{H}), 2.04(\mathrm{~s}, 3 \mathrm{H}) .{ }^{13} \mathrm{C} \mathrm{NMR}$ $\left(\mathrm{CD}_{2} \mathrm{Cl}_{2}, 75 \mathrm{MHz}\right): \delta(\mathrm{ppm}) 170.8,170.2,170.0,157.4,150.9,135.8,135.0,113.1,88.6$ 81.8, 73.6, 71.4, 71.2, 63.1, 21.0, 20.9, 20.7. HRFABMS: calcd. for $\mathrm{C}_{16} \mathrm{H}_{17} \mathrm{~N}_{6} \mathrm{O}_{7}(\mathrm{M}+$ H) ${ }^{+}$405.1159, obsd. 405.1154.

$2^{\prime}, 3^{\prime}, 5^{\prime}$-Tri- $\boldsymbol{O}$-acetyl-8-azaadenosine (6) was prepared following a previously reported procedure. $^{2}$

8-Azaadenosine ribonucleoside (7) was prepared following a previously reported procedure. $^{2}$

2',3',5'-Tris- $O$-(tert-butyldimethylsilyl)-8-azaadenosine (8). To a solution of 8azaadenosine (100 mg, $0.37 \mathrm{mmol})$ and imidazole $(203.0 \mathrm{mg}, 2.98 \mathrm{mmol})$ in DMF (400 $\mu \mathrm{L})$ was added TBDMSCl $(224.8 \mathrm{mg}, 1.49 \mathrm{mmol})$ and mixture was stirred at room temperature for $24 \mathrm{~h}$. The resulting solution was concentrated in vacuo to dryness and crude product was purified by flash column chromatography (Hexanes/Ethyl ether 1:1) to provide a white solid $(136.7 \mathrm{mg}, 60 \%) ; \mathrm{mp}=196-198{ }^{\circ} \mathrm{C} .{ }^{1} \mathrm{H} \mathrm{NMR}\left(\mathrm{CD}_{2} \mathrm{Cl}_{2}, 300 \mathrm{MHz}\right)$ : $\delta(\mathrm{ppm}) 8.43(\mathrm{~s}, 1 \mathrm{H}), 6.54$ (brs, $2 \mathrm{H}), 6.30(\mathrm{~d}, J=5.13 \mathrm{~Hz}, 1 \mathrm{H}), 5.17-5.14(\mathrm{~m}, 1 \mathrm{H}), 4.61-$ $4.59(\mathrm{~m}, 1 \mathrm{H}), 4.17-4.12(\mathrm{~m}, 1 \mathrm{H}), 3.92-3.71(\mathrm{~m}, 2 \mathrm{H}), 0.97(\mathrm{~s}, 9 \mathrm{H}), 0.85(\mathrm{~s}, 9 \mathrm{H}), 0.79$ (s, 9 
$\mathrm{H}), 0.17(\mathrm{~s}, 3 \mathrm{H}), 0.17(\mathrm{~s}, 3 \mathrm{H}), 0.01(\mathrm{~s}, 3 \mathrm{H}),-0.01(\mathrm{~s}, 3 \mathrm{H}),-0.02(\mathrm{~s}, 3 \mathrm{H}),-0.24(\mathrm{~s}, 3 \mathrm{H}) .{ }^{13} \mathrm{C}$ $\operatorname{NMR}\left(\mathrm{CD}_{2} \mathrm{Cl}_{2}, 75 \mathrm{MHz}\right): \delta(\mathrm{ppm}) 157.5,156.5,150.4,125.2,90.1,86.6,75.0,73.0,63.2$, 26.2, 26.2, 26.0, 18.7, 18.5, 18.3, -4.1, -4.3, -4.8, -5.3. HRFABMS: calcd. for $\mathrm{C}_{27} \mathrm{H}_{55} \mathrm{~N}_{6} \mathrm{O}_{4} \mathrm{Si}_{3}(\mathrm{M}+\mathrm{H})^{+}$611.3593, obsd. 611.3620.

2',3',5'-Tris-O-(tert-butyldimethylsilyl)-6-bromo-8-azanebularine (9). 2',3',5'-Tris$O$-(tert-butyldimethylsilyl)-8-azaadenosine (200 $\mathrm{mg}, 0.33 \mathrm{mmol}$ ) was dissolved in freshly distilled acetonitrile $(20 \mathrm{~mL})$ and cooled to $0{ }^{\circ} \mathrm{C}$. $t$ BuONO $(388.9 \mu \mathrm{L}, 3.27 \mathrm{mmol})$ and TMSBr $(84.7 \mu \mathrm{L}, 0.65 \mathrm{mmol})$ were added consequently and reaction mixture was kept from 0 to $+5{ }^{\circ} \mathrm{C}$ for $1 \mathrm{~h}$. The resulting solution was diluted with EtOAc/Hexanes 7: 3 (8 $\mathrm{mL})$ and washed with $5 \% \mathrm{NaHCO}_{3}(1 \times 8 \mathrm{~mL})$ and brine $(1 \times 8 \mathrm{~mL})$. The organic portion was dried over $\mathrm{Na}_{2} \mathrm{SO}_{4}$, filtered, and concentrated under reduced pressure. Purification by flash column chromatography $\left(\mathrm{CH}_{2} \mathrm{Cl}_{2}\right)$ gave a clear oil $(112.6 \mathrm{mg}, 51 \%) .{ }^{1} \mathrm{H}$ NMR $\left(\mathrm{CD}_{2} \mathrm{Cl}_{2}, 300 \mathrm{MHz}\right): \delta(\mathrm{ppm}) 8.88(\mathrm{~s}, 1 \mathrm{H}), 6.40(\mathrm{~d}, J=4.9 \mathrm{~Hz}, 1 \mathrm{H}), 5.15(\mathrm{t}, J=4.6 \mathrm{~Hz}$, $1 \mathrm{H}), 4.62(\mathrm{t}, J=3.9 \mathrm{~Hz}, 1 \mathrm{H}), 4.18(\mathrm{dd}, J=8.3,4.1 \mathrm{~Hz}, 1 \mathrm{H}), 3.89-3.72(\mathrm{~m}, 2 \mathrm{H}), 0.97(\mathrm{~s}, 9$ H), 0.83 (s, $9 \mathrm{H}), 0.77$ (s, $9 \mathrm{H}), 0.17$ (s, 3H), 0.17 (s, 3H), 0.00 (s, 3H), -0.01 (s, 3H), 0.03 (s, 3H), -0.25 (s, $3 \mathrm{H}) .{ }^{13} \mathrm{C}$ NMR $\left(\mathrm{CD}_{2} \mathrm{Cl}_{2}, 75 \mathrm{MHz}\right): \delta(\mathrm{ppm}) 156.1,149.7,146.5$, $137.4,90.7,87.0,75.4,72.8,63.0,26.2,26.1,25.9,18.7,18.5,18.3,-4.1,-4.2,-4.3,-4.8$, -5.3. HRFABMS: calcd. for $\mathrm{C}_{27} \mathrm{H}_{53} \mathrm{~N}_{5} \mathrm{O}_{4} \mathrm{Si}_{3} \mathrm{Br}(\mathrm{M}+\mathrm{H})^{+}$674.2589, obsd. 674.2611.

2',3',5'-Tris-O-(tert-butyldimethylsilyl)-6-acyloxymethyl-8-azanebularine (11). Zinc dust $(85.2 \mathrm{mg}, 1.30 \mathrm{mmol})$ in THF $(300 \mu \mathrm{L})$ was pre-activated with dibromoethane (6 $\mu \mathrm{L})$ and TMS-Cl $(5.3 \mu \mathrm{L})$ under argon atmosphrere. To the resulting suspension was added a solution of bromomethylacetate $(64.5 \mu \mathrm{L}, 0.66 \mathrm{mmol})$ in THF $(550 \mu \mathrm{L})$ and mixture was allowed to stir for $1 \mathrm{~h}$ at $15^{\circ} \mathrm{C}$. The solution of acetoxymethylzinc bromide 
$10(850 \mu \mathrm{L}, \mathrm{c}=0.07 \mathrm{mmol} / \mu \mathrm{L})$ in $\mathrm{THF}$ was transferred at room temperature to $2^{\prime}, 3^{\prime}, 5^{\prime}-$ tris-O-(tert-butyldimethylsilyl)-6-bromo-8-azanebularine $(100 \mathrm{mg}, 0.15 \mathrm{mmol})$ and $\mathrm{Pd}\left(\mathrm{PPh}_{3}\right)_{4}(8.5 \mathrm{mg}, 5 \%)$ in THF $(296 \mu \mathrm{l})$ under argon atmosphere. The resulting mixture was allowed to stir for $9 \mathrm{~h}$ at room temperature, then quenched with $1 \mathrm{M} \mathrm{NH}_{4} \mathrm{Cl}(9 \mathrm{~mL})$ and extracted with EtOAc $(3 \times 8 \mathrm{~mL})$. The combined organic layers were dried over $\mathrm{Na}_{2} \mathrm{SO}_{4}$, filtered, and concentrated under reduced pressure. The crude material was purified by flash column chromatography (Hexanes/EtOAc 8:1) to afford product as a yellow oil (76.2 mg, 77\%). ${ }^{1} \mathrm{H}$ NMR $\left(\mathrm{CD}_{2} \mathrm{Cl}_{2}, 300 \mathrm{MHz}\right)$ : $\delta(\mathrm{ppm}) 9.1$ (s, $\left.1 \mathrm{H}\right), 6.43(\mathrm{~d}, J$ $=4.9 \mathrm{~Hz}, 1 \mathrm{H}), 5.71(\mathrm{~s}, 2 \mathrm{H}), 5.14(\mathrm{t}, J=4.6 \mathrm{~Hz}, 1 \mathrm{H}), 4.66-4.63(\mathrm{~m}, 1 \mathrm{H}), 4.17(\mathrm{dd}, J=9$, 4.1 Hz, 1H), 3.90-3.72 (m, 2H), 2.24 (s, 3H), 0.97 (s, 9 H), 0.82 (s, $9 \mathrm{H}), 0.77$ (s, $9 \mathrm{H})$, 0.18 (s, 3H), 0.17 (s, 3H), -0.01 (s, 3H), -0.02 (s, 3H), -0.04 (s, 3H), $-0.25(\mathrm{~s}, 3 \mathrm{H}) .{ }^{13} \mathrm{C}$ NMR $\left(\mathrm{CD}_{2} \mathrm{Cl}_{2}, 75 \mathrm{MHz}\right): \delta(\mathrm{ppm}) 171.2,160.7,156.4,150.3,134.3,90.2,86.6,75.3$, 72.8, 63.6, 63.0, 26.2, 26.1, 25.9, 21.1, 18.7, 18.5, 18.3, -4.1, -4.3, -4.8, -5.3. HRFABMS: calcd. for $\mathrm{C}_{30} \mathrm{H}_{58} \mathrm{~N}_{5} \mathrm{O}_{6} \mathrm{Si}_{3}(\mathrm{M}+\mathrm{H})^{+}$668.3696, obsd. 668.3690 .

2',3',5'-Tris- $O$-(tert-butyldimethylsilyl)-6-mercapto-8-azanebularine (12). To a solution of 2',3',5'-Tris-O-(tert-butyldimethylsilyl)-6-bromo-8-azanebularine (129 mg, $0.19 \mathrm{mmol})$ in freshly distilled ethanol $(4 \mathrm{~mL})$ was added thiourea $(667 \mathrm{mg}, 8.75 \mathrm{mmol})$ and reaction mixture was reacted at reflux for approximately $15 \mathrm{~h}$. The resulting solution was concentrated in vacuo to dryness and crude product was purified by flash column chromatography $\left(\mathrm{CH}_{2} \mathrm{Cl}_{2}\right)$ to give an yellow oil $(61 \mathrm{mg}, 51 \%)$. ${ }^{1} \mathrm{H}$ NMR $\left(\mathrm{CD}_{2} \mathrm{Cl}_{2}, 300\right.$ MHz): $\delta$ (ppm) 8.63 (s, 1H), 8.46-843 (brm, 1H), $6.20(\mathrm{dd}, J=9,5.9 \mathrm{~Hz}, 1 \mathrm{H}), 4.46-4.43$ (m, 1 H), 4.26-4.25 (brm, 1H), 4.16-4.13 (m, 1H), 3.75-3.61 (m, 2 H), 0.99 (s, 9 H), 0.94 (s, $9 \mathrm{H}), 0.82(\mathrm{~s}, 9 \mathrm{H}), 0.23(\mathrm{~s}, 3 \mathrm{H}), 0.16(\mathrm{~s}, 3 \mathrm{H}), 0.11(\mathrm{~s}, 3 \mathrm{H}), 0.11(\mathrm{~s}, 3 \mathrm{H}), 0.10(\mathrm{~s}, 3 \mathrm{H})$, 
0.03. ${ }^{13} \mathrm{C}$ NMR $\left(\mathrm{CD}_{2} \mathrm{Cl}_{2}, 75 \mathrm{MHz}\right): \delta(\mathrm{ppm}) 181.6,169.7,157.9,157.1,137.9,87.3,81.5$, $75.0,72.0,64.1,26.3,26.2,26.0,18.8,18.6,18.5,-4.4,-4.5,-4.6,-4.7,-5.1,-5.3$. HRFABMS: calcd. for $\mathrm{C}_{27} \mathrm{H}_{54} \mathrm{~N}_{5} \mathrm{O}_{4} \mathrm{SSi}_{3}(\mathrm{M}+\mathrm{H})^{+}$628.3205, obsd. 628.3188 .

6-Methyl-8-azanebularine (13). To 2',3',5'-tri-O-acetyl-6-methyl-8-azanebularine (169.3 $\mathrm{mg}, 0.43 \mathrm{mmol})$ was added solution of methanol saturated with $\mathrm{NH}_{3}(1.9 \mathrm{~mL})$ and mixture was kept at $4{ }^{\circ} \mathrm{C}$ for $17 \mathrm{~h}$. The resulting solution was concentrated under reduced pressure, pre-adsorbed on silica gel and then purified by flash column chromatography $\left(10 \% \mathrm{CH}_{3} \mathrm{OH} / \mathrm{CH}_{2} \mathrm{Cl}_{2}\right)$ to afford a slightly yellow solid $(94.0 \mathrm{mg}, 82 \%): \mathrm{mp}=101-103$ ${ }^{\circ} \mathrm{C}{ }^{1} \mathrm{H}$ NMR $\left(\mathrm{CD}_{3} \mathrm{OD}, 300 \mathrm{MHz}\right): \delta(\mathrm{ppm}) 8.99(\mathrm{~s}, 1 \mathrm{H}), 6.45(\mathrm{~d}, J=4.4 \mathrm{~Hz}, 1 \mathrm{H}), 5.00-$ $4.97(\mathrm{~m}, 1 \mathrm{H}), 4.58-4.55(\mathrm{~m}, 1 \mathrm{H}), 4.20-4.16(\mathrm{~m}, 1 \mathrm{H}), 3.84-3.66(\mathrm{~m}, 2 \mathrm{H}), 2.98(\mathrm{~s}, 3 \mathrm{H}) .{ }^{13} \mathrm{C}$ NMR ( $\left.\mathrm{CD}_{3} \mathrm{OD}, 75 \mathrm{MHz}\right): \delta$ (ppm) 164.9, 157.0, 150.0, 137.2, 92.3, 87.8, 75.4, 72.5, 63.5, 20.2. HRFABMS: calcd. for $\mathrm{C}_{10} \mathrm{H}_{14} \mathrm{~N}_{5} \mathrm{O}_{4}(\mathrm{M}+\mathrm{H})^{+} 268.1047$, obsd. 268.1050 . 5'-O-(4,4'-Dimethoxytrityl)-6-methyl-8-azanebularine (14). Anhydrous pyridine (212 $\mu \mathrm{L}, 2.63 \mathrm{mmol}$ ), 4,4'-dimethoxytrityl chloride (163 mg, $0.48 \mathrm{mmol}$ ), and $\mathrm{AgNO}_{3}(82 \mathrm{mg}$, $0.48 \mathrm{mmol})$ were added in that order to a solution of 6-methyl-8-azanebularine $(117 \mathrm{mg}$, $0.44 \mathrm{mmol})$ in freshly distilled THF $(5 \mathrm{~mL})$. The reaction was allowed to progress at room temperature for $17 \mathrm{~h}$. The resulting solution was diluted with EtOAc $(8 \mathrm{~mL})$, filtered, and washed with saturated aqueous $\mathrm{NaHCO}_{3}(1 \times 13 \mathrm{~mL})$. The organic portion was dried over $\mathrm{Na}_{2} \mathrm{SO}_{4}$, filtered, and concentrated under reduced pressure. Purification of crude product by flash column chromatography $\left(\mathrm{CH}_{2} \mathrm{Cl}_{2} / \mathrm{CH}_{3} \mathrm{OH} / \mathrm{Et}_{3} \mathrm{~N}\right.$ 98:1:1) afforded an orange foam (173 mg, 70\%). ${ }^{1} \mathrm{H}$ NMR $\left(\mathrm{CD}_{2} \mathrm{Cl}_{2}, 300 \mathrm{MHz}\right): \delta(\mathrm{ppm}) 8.95$ (s, 1H), 7.36-7.17 (m, 9H), 6.75-6.71 (m, 4H), $6.52(\mathrm{~s}, J=3.2 \mathrm{~Hz}, 1 \mathrm{H}), 5.17-5.14(\mathrm{~m}, 1 \mathrm{H})$, $4.80(\mathrm{t}, J=5.4 \mathrm{~Hz}, 1 \mathrm{H}), 4.29(\mathrm{dd}, J=9.5 \mathrm{~Hz}, 4.9 \mathrm{~Hz}, 1 \mathrm{H}), 3.75(\mathrm{~s}, 6 \mathrm{H}), 3.40-3.24(\mathrm{~m}$, 
2H), $3.0(\mathrm{~s}, 1 \mathrm{H}) .{ }^{13} \mathrm{C} \mathrm{NMR}\left(\mathrm{CD}_{2} \mathrm{Cl}_{2}, 75 \mathrm{MHz}\right): \delta(\mathrm{ppm}) 163.9,159.1,156.6,149.2,145.4$, $136.3,136.2,130.5,128.5,128.3,127.2,113.5,90.4,86.8,84.5,74.6,72.3,64.1,55.7$, 20.7. HRFABMS: calcd. for $\mathrm{C}_{31} \mathrm{H}_{32} \mathrm{~N}_{5} \mathrm{O}_{6}(\mathrm{M}+\mathrm{H})^{+}$570.2353, obsd. 570.2329.

\section{5'-O-(4,4'-Dimethoxytrityl)-2'-O-(tert-butyldimethylsilyl)-6-methyl-8-azanebularine}

(15). To the solution of 5'-O-(4,4'-dimethoxytrityl)-6-methyl-8-azanebularine (141 mg, $0.25 \mathrm{mmol})$ in freshly distilled THF $(3 \mathrm{~mL})$ were added triethylamine $(66 \mu \mathrm{L}, 0.47 \mathrm{mmol})$ and TBDMSCl (41 mg, $0.27 \mathrm{mmol})$. The resulting solution was stirred for $5 \mathrm{~min}$ followed by addition of $\mathrm{AgNO}_{3}(46 \mathrm{mg}, 0.27 \mathrm{mmol})$. The mixture was allowed to react for approximately $24 \mathrm{~h}$ at room temperature then it was diluted with EtOAc $(9 \mathrm{~mL})$, filtered, and washed with saturated aqueous $\mathrm{NaHCO}_{3}(1 \times 11 \mathrm{~mL})$. The organic portion was dried over $\mathrm{Na}_{2} \mathrm{SO}_{4}$, filtered, and concentrated under reduced pressure. The crude material was purified by flash column chromatography (EtOAc/Hexanes 1:7) on silica gel to afford 2'-O-TBDMS product as a white foam $(54 \mathrm{mg}, 32 \%)$. The 5 '- $O$-(4,4'dimethoxytrityl)-3'-O-(tert-butyldimethylsilyl)-6-methyl-8-azanebularine was subjected to the methanol solution with traces of triethylamine for equilibration and re-purified by flash column chromatography (EtOAc/Hexanes 1:7) to give an additional amount of the 2'-O-TBDMS compound. The total yield of 2'-O-TBDMS protected nucleoside was 101 mg (60\%). ${ }^{1} \mathrm{H}$ NMR $\left(\mathrm{CD}_{2} \mathrm{Cl}_{2}, 300 \mathrm{MHz}\right): \delta(\mathrm{ppm}) 8.94(\mathrm{~s}, 1 \mathrm{H}), 7.43-7.16(\mathrm{~m}, 9 \mathrm{H}), 6.76-$ $6.73(\mathrm{~m}, 4 \mathrm{H}), 6.46(\mathrm{~d}, J=4.8 \mathrm{~Hz}, 1 \mathrm{H}), 4.48-4.43(\mathrm{~m}, 1 \mathrm{H}), 4.27(\mathrm{dd}, J=8.5 \mathrm{~Hz}, 4 \mathrm{~Hz}$, 1H), $3.75(\mathrm{~s}, 6 \mathrm{H}), 3.43-3.21(\mathrm{~m}, 2 \mathrm{H}), 3.01(\mathrm{~s}, 3 \mathrm{H}), 2.67(\mathrm{~d}, J=5 \mathrm{~Hz}, 1 \mathrm{H}), 0.82(\mathrm{~s}, 9 \mathrm{H})$,

$0.00(\mathrm{~s}, 3 \mathrm{H}),-0.17$ (s, 3H). ${ }^{13} \mathrm{C} \mathrm{NMR}\left(\mathrm{CD}_{2} \mathrm{Cl}_{2}, 75 \mathrm{MHz}\right): \delta$ (ppm) 163.9, 159.1, 156.7, $149.5,145.5,136.5,136.3,130.6,128.6,128.3,127.2,113.5,90.3,86.8,85.3,75.3,72.4$, 
64.2, 55.7, 25.8, 20.7, 18.3, -4.72, -4.81. HRFABMS: calcd. for $\mathrm{C}_{37} \mathrm{H}_{46} \mathrm{~N}_{5} \mathrm{O}_{6} \mathrm{Si}(\mathrm{M}+\mathrm{H})^{+}$ 684.3218, obsd. 684.3198 .

5'-O-(4,4'-Dimethoxytrityl)-3'-O-[(2-cyanoethoxy $)(N, N-$

\section{diisopropylamino)phosphino]-2'-O-(tert-butyldimethylsilyl)-6-methyl-8-}

azanebularine (16). To the solution of 5'-O-(4,4'-Dimethoxytrityl)-2'-O-(tertbutyldimethylsilyl)-6-methyl-8-azanebularine $(50 \mathrm{mg}, 0.07 \mathrm{mmol})$ in freshly distilled THF $(0.43 \mathrm{~mL})$ was added $N, N$-diisopropylethylamine ( $76 \mu \mathrm{L}, 0.44 \mathrm{mmol})$ followed by 2-cyanoethyl-( $N, N$-diisopropylamino)chlorophosphite $(33 \mu \mathrm{L}, 0.15 \mathrm{mmol})$. The resulting mixture was stirred at room temperature for $8 \mathrm{~h}$, then diluted with EtOAc $(10 \mathrm{~mL})$ and washed with $5 \%(\mathrm{w} / \mathrm{v})$ aqueous $\mathrm{NaHCO}_{3}(2 \times 5 \mathrm{~mL})$. The organic portion was dried over $\mathrm{Na}_{2} \mathrm{SO}_{4}$, filtered, and concentrated under reduced pressure. The crude material was purified by flash column chromatography on silica gel (EtOAc/Hexanes/Et ${ }_{3} \mathrm{~N}$ 10:89:1) to yield a white foam (52 mg, 85\%). ${ }^{31} \mathrm{P}$ NMR (121 MHz, $\mathrm{CH}_{2} \mathrm{Cl}_{2}, 85 \% \mathrm{H}_{3} \mathrm{PO}_{4}$ as external standard): $\delta(\mathrm{ppm})$ 150.2, 148.4. HRFABMS: calcd. for $\mathrm{C}_{46} \mathrm{H}_{63} \mathrm{~N}_{7} \mathrm{O}_{7} \mathrm{PSi}(\mathrm{M}+\mathrm{H})^{+}$ 884.4297, obsd. 884.4365.

\section{Deprotection and purification of 6-methyl-8-azanebularine-containing RNA}

oligonucleotides. The RNA oligonucleotide was generated using solid phase synthesis and 2'-O-TBDMS protected $\beta$-cyanoethyl phosphoramidites following a previously reported procedure. ${ }^{3}$ The CPG-bound oligonucleotide was treated with $\sim 3 \mathrm{~mL}$ of fresh solution of ammonium hydroxide in ethanol (3:1) for approximately 2 hours at room temperature and at $55{ }^{\circ} \mathrm{C}$ for approximately 5 hours. The oligo was collected and lyophilized on a Speed-Vac concentrator. The dried pellet was treated with $1 \mathrm{~mL}$ of neat triethylamine trihydrofluoride and allowed to stand for $12 \mathrm{~h}$ at room temperature. To this 
mixture was added water followed by $n$-butanol for precipitation. The crude precipitate was dissolved in 0.1 M TEA-Acetate, applied to oligonucleotide purification cartridge (Glen Research), washed with water then eluted with $20 \%$ acetonitrile/water and dried by lyophilization. The crude oligonucleotide was purified by polyacrylamide gel electrophoresis as described. ${ }^{1}$ MALDI analysis: calcd. for 6-Me-8azaN-27mer RNA oligonucleotide (M-H): 8744.3; obsd.: 8745.0.

General Biochemical Procedures. Biochemical reagents were purchased from Sigma/Aldrich unless otherwise noted. Common enzymes were obtained from Roche, Promega, or New England Biolabs. $\left[\gamma_{-}{ }^{32} \mathrm{P}\right] \mathrm{ATP}(6000 \mathrm{Ci} / \mathrm{mmol})$ was purchased from Perkin-Elmer Life Sciences. Distilled, deionized water was used for all aqueous reactions and dilutions. Storage phosphor autoradiography was performed using Eastman Kodak Co imaging plates. Visualizing of phosphor imaging plates was performed on a Molecular Dynamics Typhoon 9400. ImageQuant 5.2 software (Molecular Dynamics) was employed to evaluate data from phosphor imaging plates. An active deletion mutant of ADAR2: (MS(H) ${ }_{10}$ ENLYFQG-hADAR2 $\mathrm{a}_{216-701}$ ) was overexpressed in Saccharomyces cerevisiae and purified as previously described. ${ }^{1,4}$

Preparation of Duplex Structure. The RNA oligonucleotide containing 6-Me-8azaN was 5'-end-labeled and then hybridized to its unlabeled complement strand by following a previously reported procedure. ${ }^{1}$

Gel Mobility Shift Assay. The dissociation constant for 6-Me-8azaN 27-mer RNA duplex binding to the editing enzyme was determined following a previously reported procedure. ${ }^{1}$ 


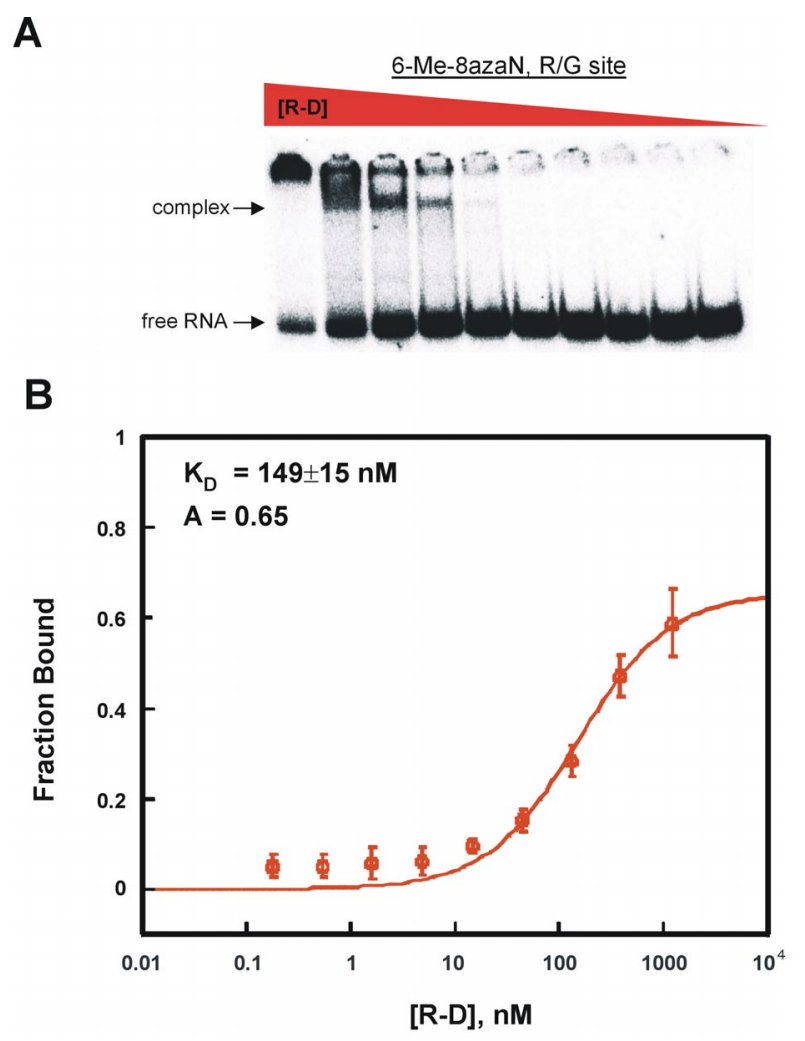

Figure 1. Gel mobility shift experiments to measure the binding of an active deletion mutant of ADAR2 to 6-Me-8azaN 27-mer RNA duplex. A. Storage phosphor autodiogram of native polyacrylamide gel separating bound RNA from free. B. The plot of fraction of RNA bound as a function of protein concentration.

\section{References:}

(1) Haudenschild, B. L.; Maydanovych, O.; Veliz, E. A.; Macbeth, M. R.; Bass, B. L.; Beal, P. A. J. Am. Chem. Soc. 2004, 126, 11213-11219.

(2) Seela, F.; Munster, I.; Lochner, U.; Rosemeyer, H. Helv. Chim. Act. 1998, 81, 11391155.

(3) Veliz, E. A.; Easterwood, L. M.; Beal, P. A. J. Am. Chem. Soc. 2003, 125, 1086710876.

(4) Macbeth, M. R.; Lingam, A. T.; Bass, B. L. RNA 2004, 10, 1563-1571. 